\title{
Conversion to Reverse Total Shoulder Arthroplasty with and without Humeral Stem Retention: Mid-to Long-Term Results
}

Marios Loucas*, Rafael Loucas, Philipp Kriechling, Samy Bouaicha and Karl Wieser

Department of Orthopedics, Balgrist University Hospital, Zurich, Switzerland

\begin{abstract}
Background: Over the past decade, conversion to Reverse Total Shoulder Arthroplasty (RTSA) has become the preferred treatment for revision of an Anatomic Hemi (HA) or Total Shoulder Arthroplasty (TSA). However, conversion of failed stemmed shoulder arthroplasty to RTSA is still a highly demanding procedure and carries unique technical challenges and risks.
\end{abstract}

Questions/Purposes: This study aimed to analyze the mid- to long-term results after conversion of failed anatomical shoulder arthroplasty to RTSA and investigate whether preserving the humeral stem offers advantages over revising the humeral stem.

Materials and Methods: Between 2005 and 2018, 99 hemiarthroplasties and 62 total shoulder arthroplasties (total $=161$ shoulders; 157 patients) were revised to RTSA without $(n=47)$ or with $(n=114)$ stem exchange. Complications and revisions were documented from medical and surgical records. Longitudinal pre- and post-operative clinical (Constant-Murley (CS) score, Subjective Shoulder Value (SSV)), and radiographic outcomes were assessed. Complete clinical and radiographic follow-up was available on $80 \%$ of shoulders (127 patients; 128 of 161 procedures, 46 without and 82 with stem exchange) at a minimum of 24 months and a mean of 70 months (range, 24 to 184 months).

OPEN ACCESS

${ }^{*}$ Correspondence:

Marios Loucas, Department of

Orthopedics, Balgrist University

Hospital, Zurich, Forchstrasse 340,

CH-8008 Zurich, Switzerland, Tel: +41

4451073 78;

E-mail:marios.loucas@hotmail.com

Received Date: 12 Jul 2021

Accepted Date: 27 Jul 2021

Published Date: 10 Aug 2021

Citation:

Loucas M, Loucas R, Kriechling P, Bouaicha S, Wieser K. Conversion to Reverse Total Shoulder Arthroplasty with and without Humeral Stem Retention: Mid-to Long-Term Results. Clin Surg. 2021; 6: 3277.

Copyright $\odot 2021$ Marios Loucas. This is an open access article distributed under the Creative Commons Attribution License, which permits unrestricted use, distribution, and reproduction in any medium, provided the original work is properly cited.
Results: Humeral stem retention was associated with a significantly reduced surgical time (193 min vs. $227 \mathrm{~min}, \mathrm{p}=0.001$, less blood loss $(591 \mathrm{~mL}$ vs. $753 \mathrm{~mL}, \mathrm{p}=0.037)$, less intraoperative complications (13\% vs. 19\%; Odds Ratio (OR), 1.4, $\mathrm{p}=0.32$ ) and fewer subsequent reinterventions (19\% vs. 28\%; $\mathrm{OR}, 2.3, \mathrm{p}=0.06$ ). The complication/revision rate leading to drop out from the study was considerable in the stem revision group (ten patients; ten of 114 shoulders (9\%)), but there were no complicationrelated dropouts in the stem-retaining group.

Conclusion: Our findings suggest that humeral stem revision is associated with decreased surgical time, less blood loss, less intra- and postoperative complications, and a lower revision rate compared to humeral stem retention. Based on these findings, a shoulder arthroplasty system modularity offers substantial benefit if conversion to reverse total shoulder arthroplasty becomes necessary.

Level of Evidence: Level III, therapeutic study.

Keywords: Reverse total shoulder arthroplasty; Revision shoulder arthroplasty; Failed shoulder arthroplasty; Hemiarthroplasty; Anatomic total shoulder arthroplasty; Conversion

\section{Introduction}

Over the past thirteen years, there has been an increase in the number of revision shoulder arthroplasties [1-5]. The most common reasons for failure are implant loosening, infection, instability, glenoid wear, or rotator cuff dysfunction. These can cause serious challenges [6-11]. Due to its design, which addresses prosthetic instability and rotator cuff dysfunction, RTSA has become the favored treatment for failed anatomic HA or TSA, with favorable short- and mid-term outcomes reported in multiple studies [3,12-20]. However, conversion of failed shoulder arthroplasty to RTSA is still a technically challenging and demanding procedure with a considerable risk of intra- and postoperative complications and a $22 \%$ to $32 \%$ revision rate $[15,17,20-22]$. Previous studies on the procedures have indicated that in particular, revision of a well-fixed humeral stem was associated with increased blood loss and operative time, as well as with increased intraoperative complications such as the high risk of loss of humeral bone stock, nerve injury, periprosthetic fracture, and malunion 
or nonunion of a humeral osteotomy with later humeral component loosening $[1,17,21,23]$. This study aimed to analyze the mid- to longterm results regarding the functional outcome, patient satisfaction, complication-and reoperation rate after conversion of failed HA and TSA to RTSA. We hypothesized that conversion of failed shoulder arthroplasty to RTSA with stem retention is superior to revision with stem exchange due to reduced operative time, reduced blood loss, fewer intraoperative complications, and potentially fewer subsequent revisions.

\section{Material and Methods}

This study was approved by the ethics committee of the University of Zurich (ID 2018-01494) and conducted following the Helsinki Declaration.

\section{Patients selection}

All patients identified in our prospectively collected, comprehensive institutional RTSA database treated with aseptic single-stage revision of HA or TSA to RTSA between January 2005 and December 2018 were retrospectively reviewed. Ninety-nine shoulders underwent conversion from hemiarthroplasties and 62 from total shoulder arthroplasties to RTSAs. Thirty of the patients (33 shoulders) were not available for final clinical follow-up. Ten of these patients (all in the group with stem exchange) had complications requiring major re-revision surgery. These patients had to be excluded from the functional assessment, and their surgeries were rated as failures. Clinical and radiographic follow-up of the remaining 127 patients (128 shoulders, 82 with and 46 without stem exchange) was at a minimum of 24 months and a mean of 70 months (range, 24 to 184 months) (Figure 1). Except for the number of cemented and cementless implants between the two groups (Table 1), no statistically significant difference was found between the two study groups' demographic data. The mean age of the patients at the time of revision to RTSA was 64 years (range: 24 to 86 years). The median number of operations before conversion to RTSA was 2 (range, 1 to 8 operations). The main indication for conversion to RTSA was secondary rotator cuff failure, followed by glenoid erosion after HA or glenoid component failure after TSA (Table 2). Patients were separated into two cohorts: The retention group included 46 patients who underwent revision of a HA or anatomic TSA in which the original humeral stem was retained during the conversion to RTSA. In this group, the humeral component was determined to be well fixed, adequately positioned, and therefore not in need of revision. The exchange group included 81 patients ( 82 shoulders) who underwent revision of a HA or TSA

\section{7 patients (161 shoulders) with revision for HA/TSA}

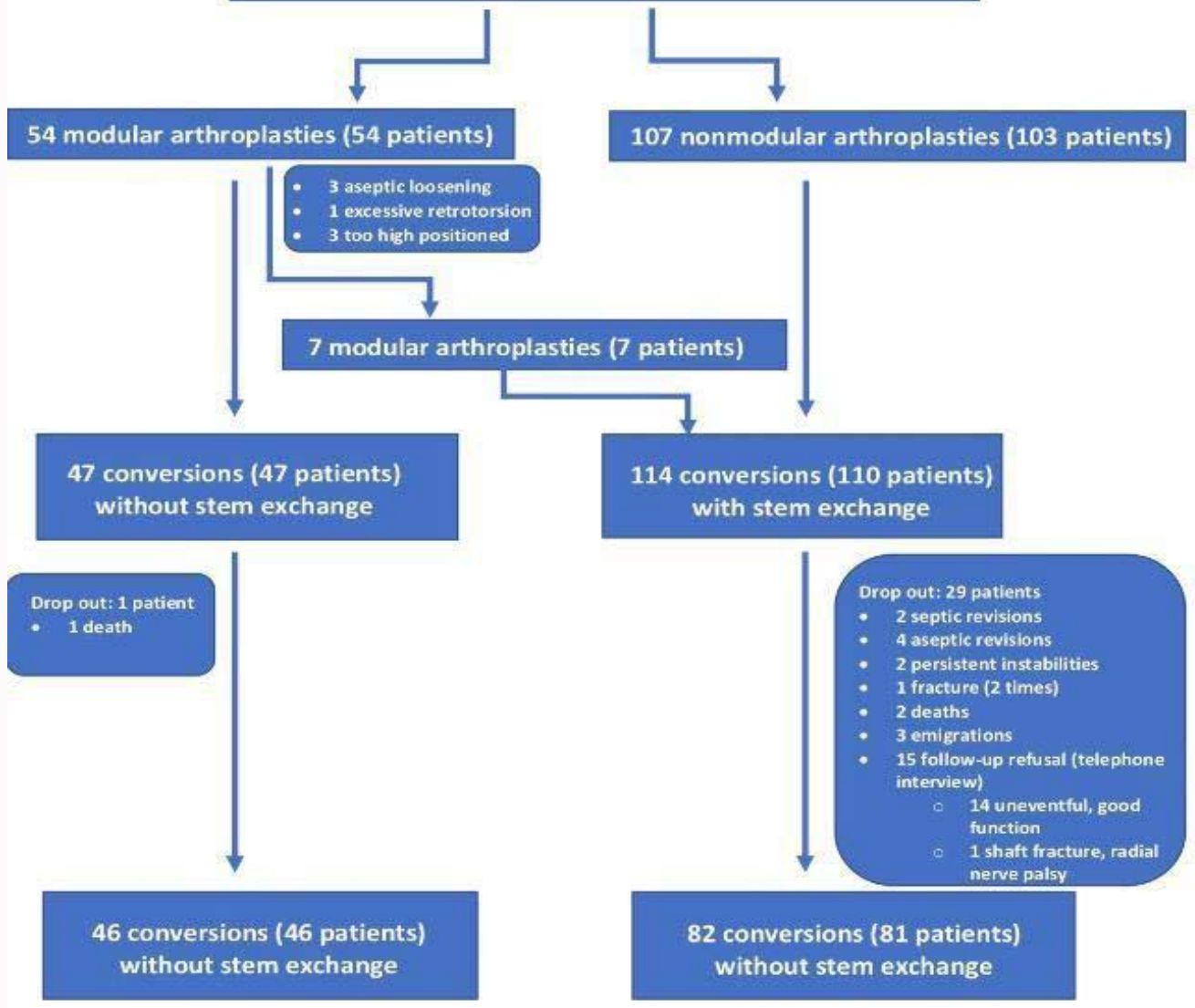

Patients with complete clinical follow-up analysis (minimum, 24 months, mean 70 months; range, 24-184 months)

Figure 1: The flow diagram shows enrollment, dropout, and loss to follow-up of the study cohort.

HA: Hemiarthroplasty; TSA: Total Shoulder Arthroplasty 
to RTSA that necessitated, either due to implant design ( $\mathrm{n}$ : 54) or due to loosening or malposition (n:28) revision of a humeral stem (Table 2). Operative time and blood loss were documented from the anesthesia records. Clinical and radiographic examinations for all patients undergoing arthroplasty in our unit were performed preoperatively and approximately 1 year, 2 to 5 years, 5 to 8 years, 8 to 10 years, and more than 10 years postoperatively. The clinical examination included measurement of active and passive ranges of motion using a handheld Goniometer and assessment of the absolute (aCS) and relative Constant Scores (rCS) and Subjective Shoulder Value (SSV) [24-26]. Abduction strength in the scapular plane was measured with a validated electronic dynamometer (Isobex; Cursor, Bern, Switzerland) [27]. Preoperatively and postoperatively, standardized true anteroposterior, axillary lateral, and scapular lateral (Neer view) radiographs were obtained for all patients. On the postoperative radiographs, inferior scapular notching according to the Sirveaux classification, radiolucency, heterotopic ossification, glenoid or humeral loosening, and glenoid or acromial fracture were evaluated. If the patient had to undergo a later revision with exchange or removal of the converted implant had become necessary, the result was treated as a failure, and this patient was excluded from functional assessment.

\section{Surgical protocol}

All revisions were performed with the patient in a beach chair position, through a deltopectoral approach, by an experienced shoulder surgeon. All patients received perioperative intravenous antibiotic prophylaxis and underwent general anesthesia in combination with an interscalene block. The subscapularis tendon was evaluated intraoperatively. If the tendon was intact, it was elevated off the lesser tuberosity and reattached before wound closure. The majority of cases 99\%, the Anatomical Shoulder Inverse/Reverse System (Zimmer Biomet, Warsaw, Indiana, USA) and in 1\% of all cases Delta III ((DePuy International, Leeds, UK) were used as the revision implants on the humeral side. For patients with a modular system $(n=46)$, conversion to RTSA was performed without removal of the humeral stem whenever feasible (23 total shoulder arthroplasties and 23 hemiarthroplasties) (Figure 1). Firstly, the prosthetic head was carefully detached from the stem using an extraction instrument, and the humeral metaphysis was prepared using a flat reamer, which allows seating of the reverse metaphysis on the humeral stem. Of the convertible anatomical stems, seven had to be converted: 3 dues to aseptic loosening, 1 because of excessive glenoid retroversion, and 3 were too high positioned. Of the remaining 76 shoulders without modular implants, ten were malpositioned and seventeen were loose. To allow conversion to RTSA, the remaining 55 stems had to be exchanged, although they were well fixed and well-positioned. In cases with well-fixed, nonconvertible stems, careful removal of the prosthesis was performed without splitting the humeral shaft and retaining as much bone stock as possible. After preparing the humeral stem, the glenoid was exposed. If the cement mantle was stable, it was left in place, cleaned with a drill, and a smaller prosthesis was cemented into the old cement mantle $(n=19)$. In the remaining 36 shoulders, 11 shoulders were press-fitted in the shaft, and in 25 cases, cement was removed. Furthermore, any remaining glenoid labrum was excised, and capsule or scar tissue was released for all conversions from HA to RTSA. Additionally, in cases where the glenoid component had to be extracted and cement removed, special care was taken to preserve as much of the glenoid bone stock as possible. The tendon of the triceps long head was released from the infra-glenoid tubercle of the scapula with careful axillary nerve protection. The Anatomical Shoulder System glenoid (Zimmer Biomet, Warsaw, Indiana, USA) component was used as the standard implant in the majority of procedures $(n=123)$. In nine cases, a long-pegged glenoid base plate was used (Aequalis Reversed II (Tornier, Amsterdam, The Netherlands); two Delta III (De Puy International, Leeds, UK)); one Affinis Inverse System (Mathys AG Bettlach Switzerland); two Lima SMR Reverse Total Shoulder Arthroplasty system (Lima Corporate, Udine- Italy) and twenty-four Trabecular metal (Zimmer Biomet, Warsaw, Indiana, USA)). The guide and subsequent guide wire were positioned so that the glenoid base plate was as low as possible with a neutral or minimal inferior tilt. In order to provide primary angular stability, locking screws were used. In notable glenoid bone loss cases, osseous autograft $(n=19)$ or allograft $(n=10)$ was used to provide the glenoid base plate's inherent stability and lateralization. The height polyethylene components were chosen based on the arms preoperatively assessed length of the arm, the soft tissue tension, and the shoulder joint stability. After the final implantation and readaptation of the subscapularis, two suction drains were inserted for $24 \mathrm{~h}$ to $48 \mathrm{~h}$. Postoperatively patients were immobilized in a sling for 4 to 6 weeks.

\section{Follow-up assessment and patient dropouts}

All included 157 patients (161 shoulders), had complete perioperative as well as early postoperative data. However, some patients had incomplete clinical and/or radiological follow-up analysis for the following reasons (Figure 1): Two patients who underwent stem exchange had a late (chronic) infection, which was treated successfully with a two-stage revision and implant exchange approximately two years after conversion to RTSA; and four patients (four shoulders) underwent revision surgery, one because of humeral stem loosening after intraoperative induced humeral shaft fracture (13 months), two for posttraumatic humeral stem loosening and one because of glenoid loosening ( 21 months). These six patients (all with stem exchange) were rated as failures but excluded from further functional analysis. Additionally, two patients with stem exchange had revision of the RTSA for instability, albeit the instability in one of them persisted. One patient had six months postoperatively an acromion fracture and sustained an additional periprosthetic shaft fracture 30 months after conversion surgery. These fractures were treated conservatively. For the patients mentioned above, no functional outcome scores were available. Three patients emigrate after conversion surgery and could not be followed, and three patients died unrelated to the shoulder surgeries. Additional fifteen patients refused follow-up visits. Fourteen of them were contacted via telephone and reported an uneventful course with good function. The last patient had an intraoperative humeral shaft fracture with radial nerve palsy and preferred further treatment at another hospital. As such, there were twenty-three patients lost to follow-up and ten complication-related dropouts without documented complications. The majority $(n=29)$ of the patients were in the exchange group and one patient in the stem retention group. Therefore, 127 patients (128 shoulders) had complete perioperative documentation and at least 2 years follow-up.

\section{Statistical analysis}

The Mann-Whitney U test was used for statistical analysis of intraoperative complications and surgical time. The two cohorts described above were compared using an independent-samples t-test for continuous variables and chi-square analyses for categorical variables to detect differences between the groups. The exchange 

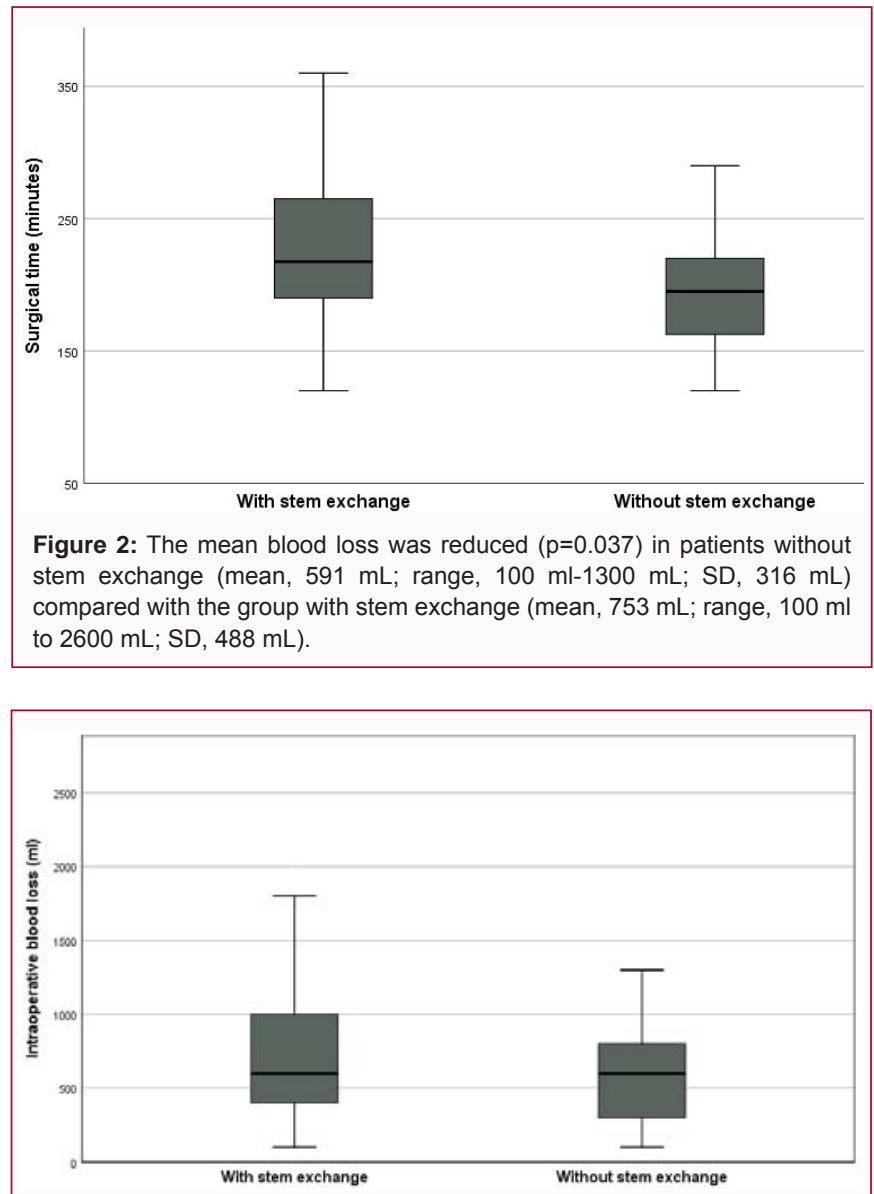

Figure 3: The surgical time could be decreased to 193 minutes (range, 75 minutes to 290 minutes; SD, 44 minutes) when the stem could be left in place, which spared, on average, 34 minutes surgical time $(p<0.0001)$ compared with cases in which the stem had to be exchanged (mean, 227 minutes; range, 120 minutes to 360 minutes; SD, 49 minutes).

and retention groups were also compared according to the index procedure (HA and TSA). A difference of $\mathrm{p}<0.05$ was considered to be statistically significant. For identifying interacting factors, Odds Ratios (OR) were calculated as crude OR and stratified by subgroups. All statistical analyses were processed with IBM SPSS statistics software (Version 25.0; Chicago, IL, USA).

\section{Results}

Patients who underwent conversion to RTSA without stem revision (retention group) had less intraoperative blood loss and operative time than those who had completed stem revision (exchange group). Blood loss averaged $591 \mathrm{~mL}$ (range, $100 \mathrm{~mL}$ to $1300 \mathrm{~mL}$; SD, $316 \mathrm{~mL}$ ) without and $753 \mathrm{~mL}$ (range, $100 \mathrm{~mL}$ to $2600 \mathrm{~mL}$; SD, 488 $\mathrm{mL}$ ) with stem exchange $(\mathrm{p}=0.037)$ (Figure 2). Surgical time averaged $193 \mathrm{~min}$ (range, $75 \mathrm{~min}$ to $290 \mathrm{~min}$; SD, $44 \mathrm{~min}$ ) without stem exchange and $227 \mathrm{~min}$ (range, $120 \mathrm{~min}$ to $360 \mathrm{~min}$; SD, $49 \mathrm{~min}$ ) with stem exchange $(\mathrm{p}=0.0001)$ (Figure 3$)$.

\section{Complications}

We identified 22 (19\%) intraoperative and 25 (21 patients (22\%)) postoperative complications in the 114 shoulders in the exchange group compared to six (13\%) intraoperative and five (11\%) postoperative complications in the 47 shoulders in the retention group. The odds ratios of sustaining an intraoperative or postoperative complication for patients with stem exchange was 1.4 (95\% CI, 0.7
Table 1: Demographic data for the study groups.

\begin{tabular}{|c|c|c|c|}
\hline Parameter & $\begin{array}{c}\text { With stem exchange } \\
\text { (81 patients [82 } \\
\text { procedures]) }\end{array}$ & $\begin{array}{c}\text { Without stem } \\
\text { exchange (46 } \\
\text { patients [46 } \\
\text { procedures]) }\end{array}$ & $\begin{array}{c}\mathbf{p} \\
\text { value }\end{array}$ \\
\hline $\begin{array}{c}\text { Age at conversion } \\
\text { (years) }\end{array}$ & $65(40-84)$ & $63(24-85)$ & 0.28 \\
\hline $\begin{array}{c}\text { Gender (female:male) } \\
\text { Interventions before } \\
\text { conversion }\end{array}$ & $75: 39: 00$ & $23: 24$ & 0.19 \\
\hline $\begin{array}{c}\text { Hemiarthroplasty: } \\
\text { Anatomic total } \\
\text { shoulder arthroplasty }\end{array}$ & $1.8(1-8)$ & $1.6(1-8)$ & 0.63 \\
\hline $\begin{array}{c}\text { Cemented humeral } \\
\text { implant }\end{array}$ & $75: 39: 00$ & $24: 23: 00$ & 0.08 \\
\hline
\end{tabular}

Table 2: Indication for conversion to RTSA.

\begin{tabular}{|c|c|c|}
\hline \multicolumn{1}{|c|}{ Indication } & \multicolumn{2}{|c|}{ Number of indications } \\
\hline & $\begin{array}{c}\text { With stem } \\
\text { exchange }\end{array}$ & $\begin{array}{c}\text { Without stem } \\
\text { exchange }\end{array}$ \\
\hline Rotator cuff deficiency/instability & 55 & 29 \\
\hline $\begin{array}{c}\text { Glenoid erosion after hemiarthroplasty } \\
\text { Failure of glenoid component after total } \\
\text { shoulder arthroplasty }\end{array}$ & 11 & 6 \\
\hline Aseptic stem loosening & 12 & 4 \\
\hline Stem malposition with functional deficit & 10 & 5 \\
\hline Sec. dislocation of greater tuberosity & 8 & \\
\hline Aseptic glenoid loosening & 5 & 3 \\
\hline Septic stem loosening & 6 & \\
\hline
\end{tabular}

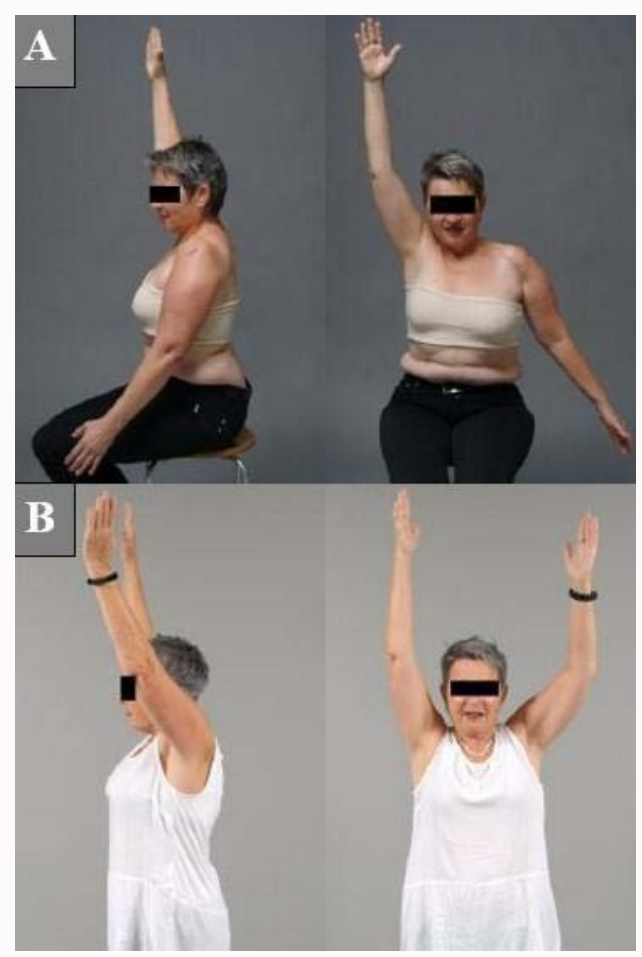

Figure 4: A 55-year-old woman presented with a severe pseudoparalysis and mal union of greater and lesser tubercles of her left arm 7 months after a hemiarthroplasty as a result of a four-part humeral head fracture. Pre- (A) and postoperative $(B)$ range of motion at ten years after conversion to a reverse total shoulder arthroplasty.

to 3.0 ) and 1.9 (95\% CI, 0.8 to 4.4 ), compared to patients without stem exchange. The exchange group had twenty-four conversionrelated reoperations (twenty patients $(20 \%)$ ), whereas in four of the 
Table 3: Complications and reoperations.

\begin{tabular}{|c|c|c|c|c|c|c|}
\hline \multirow[t]{2}{*}{ Complication } & \multicolumn{3}{|c|}{ With stem exchange } & \multicolumn{3}{|c|}{ Without stem exchange } \\
\hline & Intraoperative & Postoperative & Reoperation & Intraoperative & Postoperative & Reoperation \\
\hline Shaft fracture & 13 & $2^{*}$ & 1 & & & \\
\hline $\begin{array}{l}\text { Shaft fracture with radial } \\
\text { nerve palsy }\end{array}$ & 2 & 3 & & 1 & & \\
\hline Greater tuberosity fracture & 2 & & & 3 & & \\
\hline Glenoid fracture & 2 & & & & & \\
\hline Radial nerve palsy & 1 & & & & & \\
\hline Cement extrusion & 2 & & 2 & 1 & & \\
\hline Acromion fracture & & $5^{*}$ & 2 & & 1 & \\
\hline $\begin{array}{l}\text { Glenoid component } \\
\text { loosening }\end{array}$ & & $3^{*}$ & 2 & & 1 & \\
\hline Early infection & & $2^{*}$ & 2 & & 1 & 1 \\
\hline Wound healing problem & & 2 & 5 & & 1 & 1 \\
\hline Instability & & 2 & & & & \\
\hline Humeral stem loosening & & 2 & 2 & & & \\
\hline $\begin{array}{l}\text { Dislocation of glenoid } \\
\text { components }\end{array}$ & & $1^{*}$ & 1 & & & \\
\hline Total & 22 & 25 (21 patients) & 24 (20 patients) & 6 & 5 & 4 \\
\hline
\end{tabular}

* Four patients with two postoperative complications (one with an acromial fracture and periprosthetic humeral shaft fracture; two with an acromial fracture and glenoid component loosening and one with a traumatic humeral shaft fracture and early infection after fracture treatment)

Table 4: Clinical results according to Constant and Murley score and subjective shoulder value*.

\begin{tabular}{|c|c|c|c|c|c|c|c|c|c|}
\hline \multirow{2}{*}{$\begin{array}{l}\text { Clinical outcome } \\
\text { parameter }\end{array}$} & \multicolumn{4}{|c|}{ With stem exchange ( $n=82$ shoulders) } & \multicolumn{4}{|c|}{ Without stem exchange ( $n=46$ shoulders) } & \multirow[t]{2}{*}{ p value ${ }^{\star}$} \\
\hline & Preoperative & Follow-up & Improvement & $p$ value & Preoperative & Follow-up & Improvement & $\mathrm{p}$ value & \\
\hline $\begin{array}{l}\text { Absolute Constant and } \\
\text { Murley score }\end{array}$ & $30(6-80)$ & $52(8-86)$ & 22 & 0.0001 & $36(6-80)$ & $59(21-85)$ & 23 & 0.0001 & 0.1 \\
\hline $\begin{array}{l}\text { Adjusted Constant and } \\
\text { Murley score }\end{array}$ & $36(8-92)$ & $62(8-97)$ & 26 & 0.0001 & $44(8-93)$ & $70(28-98)$ & 26 & 0.0001 & 0.13 \\
\hline CS pain score & $7(0-15)$ & $12(3-15)$ & 5 & 0.0001 & $8(0-15)$ & $12(2-15)$ & 4 & 0.0001 & 0.62 \\
\hline Flexion $\left({ }^{\circ}\right)$ & $64(0-160)$ & $101(0-170)$ & 37 & 0.0001 & $78(0-170)$ & $109(0-175)$ & 31 & 0.0001 & 0.46 \\
\hline Abduction $\left({ }^{\circ}\right)$ & $61(0-175)$ & $102(0-170)$ & 41 & 0.0001 & $70(0-180)$ & $116(0-170)$ & 46 & 0.0001 & 0.12 \\
\hline External rotation $\left({ }^{\circ}\right)$ & $25(-10-80)$ & $12(-50-55)$ & -13 & 0.0001 & $27(0-70)$ & $18(-30-50)$ & -8 & 0.0001 & 0.25 \\
\hline $\begin{array}{l}\text { Subjective shoulder value } \\
\text { (maximum 100) }\end{array}$ & $29(0-80)$ & $56(10-100)$ & 27 & 0.0001 & $35(0-80)$ & $65(10-100)$ & 30 & 0.008 & 0.09 \\
\hline
\end{tabular}

* The data are mean with the range in parentheses; ${ }^{+}$comparison between groups

47 patients in the retention group, (9\%) reoperations were performed (OR: $2.3 ; 95 \% \mathrm{CI}, 0.9$ to 5.7 ). Therefore, the complication rate leading to dropout with more or less functional compromise was substantial in the exchange group of 110 patients (ten patients; ten of 114 shoulders (9\%)), but there were no complication-related dropouts in the stem-retaining group (Table 3 ).

\section{Clinical results}

The 46 patients in the retention group showed an improvement of the relative Constant and Murley score from $43 \%$ (range, $8 \%$ to $93 \%$ ) to $68 \%$ (range, $28 \%$ to $98 \%$ ) ( $\mathrm{p}=0.002$ ), the mean absolute Constant and Murley score improved from 36 (range, 6 to 80 points) to 58 points (range, 21 to 85 points) ( $\mathrm{p}=0.002$ ), and the mean subjective shoulder value increased from $35 \%$ (range, $0 \%$ to $80 \%$ ) to $64 \%$ (range, $10 \%$ to $100 \%)(\mathrm{p}=0.002)$. The 114 shoulders in the exchange group showed an improvement of the relative Constant and Murley score from $36 \%$ (range, $8 \%$ to $92 \%$ ) to $62 \%$ (range, $8 \%$ to $97 \%$ ) ( $\mathrm{p}=0.0001$ ), the mean absolute Constant and Murley score improved from 30 (range, 6 to 80 points) to 52 points (range, 8 to 86 points) $(\mathrm{p}=0.0001$ ), and the mean subjective shoulder value increased from $28 \%$ (range,
$0 \%$ to $70 \%)$ to $55 \%$ (range, $0 \%$ to $100 \%)(\mathrm{p}=0.002)$. There were also significant improvements in ROM, pain scores, and strength (all $\mathrm{p}<0.001)$ in both groups. In the exchange group, the mean active anterior elevation improved from $63^{\circ}$ to $100^{\circ}$ and active abduction from $60^{\circ}$ to $100^{\circ}$. In the retention group, the mean active anterior elevation improved from $77^{\circ}$ to $109^{\circ}$ and active abduction from $69^{\circ}$ to $116^{\circ}$ (Table 4 and Figure 4 ).

\section{Radiological results}

Heterotopic ossifications were found in $15 \%(n=24)$ of the cases. Of these, 7 cases were in the retention group and 17 cases in the exchange group. They did not compromise the clinical outcome. An asymptomatic radiolucency around the stem was found in one patient in the retention group. Scapular notching was noted in 43 (27\%) and did not affect the clinical outcome. Of these, 14 cases were in the retention group and 29 cases in the exchange group. According to the Sirveaux classification 29, the grade of notching was mild (I or II) in 31 patients and severe (grade III and IV) in 12 patients. The radiological results are summarized in Table 5. Regarding the radiological outcome, we did not find any significant differences 


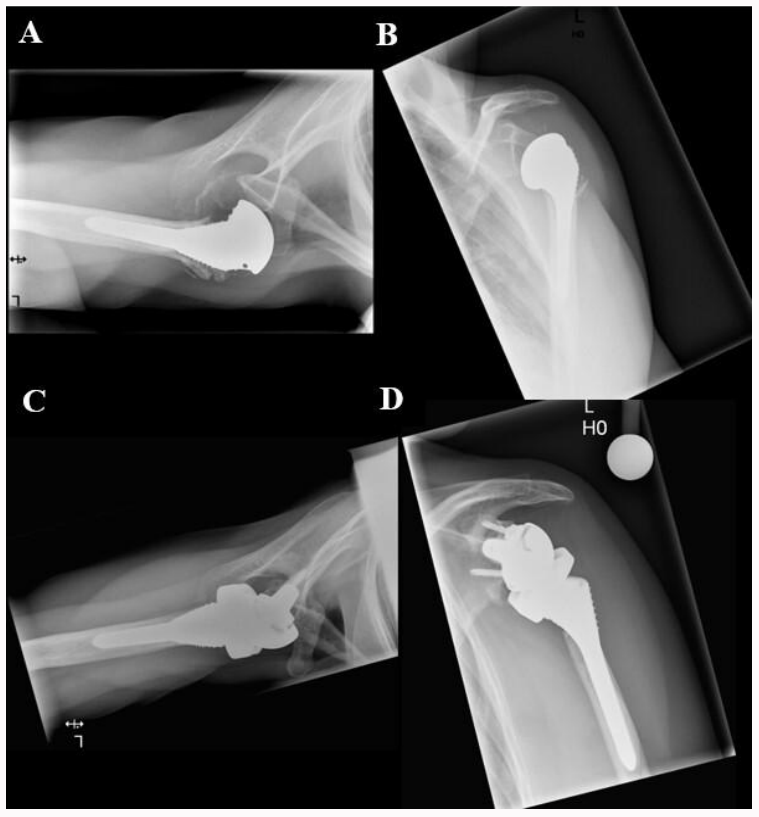

Figure 5: A 55-year-old woman presented with a severe pseudoparalysis and mal union of greater and lesser tubercles of her left arm 7 months after a hemiarthroplasty as a result of a four-part humeral head fracture. Preoperative (A) axial and (B) AP radiographs show a well-fixed humeral stem. Ten years after conversion to a reverse total shoulder arthroplasty, the patient still shows a pain-free overhead function with good component positioning with some bone resorption at the greater tuberosity but without any signs of loosening observed on the (C) AP and (D) axial radiographs.

Table 5: Radiologic results.

\begin{tabular}{|c|c|c|}
\hline Radiologic item & With stem exchange & Without stem exchange \\
\hline Scapular notching rate & $18 \%(n=29)$ & $9 \%(n=14)$ \\
\hline Grade I & $7 \%(n=12)$ & $6 \%(n=9)$ \\
\hline Grade II & $3 \%(n=4)$ & $4 \%(n=6)$ \\
\hline Grade III & $4 \%(n=7)$ & $0,6 \%(n=1)$ \\
\hline Grade IV & $1 \%(n=2)$ & $1 \%(n=2)$ \\
\hline Heterotopic ossifications & $11 \%(n=17)$ & $4 \%(n=7)$ \\
\hline Radiolucency & $0,6 \%(n=1)$ & \\
\hline
\end{tabular}

between the two groups (Figure 5).

\section{Discussion}

Our study's most important finding was that revision of stemmed anatomical hemi- or total-shoulder arthroplasties to reverse shoulder arthroplasties performed without stem exchange significantly decreased operative time and blood loss compared to those with stem exchange. Humeral stem removal has potential morbidity, including gross bone loss, damage to remaining muscular attachments, neurovascular structures, and increases surgical time. Some years ago, our group 32 performed a first retrospective casecontrol study analyzing the clinical outcomes of conversion of stemmed HA or TSA and reporting advantages of a modular stem design in a smaller patient cohort and with a shorter follow-up period. Back then, we found that stem retention resulted in $346 \mathrm{ml}$ less intraoperative blood loss and $58 \mathrm{~min}$ shorter operative time, with a decreased intraoperative complication rate $(8 \% v s .30 \%)$ and reintervention rate $(8 \%$ vs. $14 \%)$. In our current study, with almost tripled patient numbers and substantially longer follow-up period, these advantages could still be detected but to a lesser degree. Stem retention resulted in $162 \mathrm{ml}$ reduced intraoperative blood loss and spared $34 \mathrm{~min}$ of surgical time. The intraoperative and postoperative complication rate was $19 \%$ and $22 \%$ in exchange and $13 \%$ and $11 \%$ in the retention group. Compared to our current study with 99 (61\%) revised HA and $62(39 \%)$ revised TSA, our previews study back in 2015 examined 48 (86\%) HA and 8 (14\%) TSA conversions to RTSA. This higher percentage of potentially more complex revisions of TSA to RTSA may affect the differences in surgical time and blood loss compared to the current study results. The main indication for conversion to RTSAs was secondary rotator cuff failure, followed by glenoid erosion after HA or failure of the glenoid component after TSA. These indications were similarly distributed between the two groups and were consistent with the indications reported in the literature with rotator cuff failure being the most common, followed by instability and component loosening [3]. When considering the intra- and postoperative complications, our study showed that proper implantation (correct prosthetic height and torsion) of a modular humeral stem during the index operation could potentially reduce the complexity of revising a shoulder arthroplasty to RTSA. Therefore, we emphasize the importance of a well-positioned convertible-platform stem as an absolute prerequisite for stem retention $[1,28,17]$. The results after a mean of 70 months after conversion surgery to RTSA showed an improved shoulder function. A substantial pain relief, a relative Constant score of $68 \%$, a mean forward flexion of $103^{\circ}$ suggesting a satisfactory clinical outcome and range of movement, but it is less favorable than results after a primary RTSA. WeberSpickschen et al. [29] performed a retrospective review investigating the clinical efficacy of modular prosthetic system in conversion to RTSA. They reported satisfactory functional outcomes. The authors concluded that retaining the stem does not compromise the surgical outcome. Our results also show similar functional scores and range of motion between the two groups. Therefore, humeral stem retention is proven not to impair the functional outcome of the conversion procedure and provides at the same time the advantages of reduced complication and reoperation rates. Merolla et al. [30] recently evaluated the results of 157 patients who underwent conversion of a failed HA to a revision RTSA. In accordance with our findings, these authors showed significant improvements in patients' preoperative to postoperative pain and shoulder range of motion $(\mathrm{P}<0.0001)$. After conversion from a HA undertaken for fracture to RTSA in 25 patients, Gohlke et al. [12] reported a relative CS of $63 \%$ and a mean forward flexion of $125^{\circ}$ at a mean follow-up of 32 months. Furthermore, Melis et al. [20] performed a retrospective, multicenter cohort study of 34 patients who underwent revision RTSA for failed TSA. They reported a significant improvement of the Constant Murley score improved from 24 to $55(\mathrm{p}<0.0001)$ and the active anterior flexion from $68^{\circ}$ to $121^{\circ}(\mathrm{p}<0.0001)$. The postoperative complication rate was $30 \%$, and $22 \%$ of these patients needed re-revision surgery. A recent systematic review by Knowles et al. [3] comparing North American vs. European outcomes and complications in revision shoulder arthroplasty noted a total complication rate for all revisions of $17 \%$. There were 341 complications (in 2001 patients), and of those, a large number (74\%) led to a reoperation. Compared to primary shoulder replacement RTSA is associated with an up to four times increased complication rate [31]. In an additional systematic review, Zumstein et al. [5] noted a complication rate after revision RTSA of 33\%. However, Weber-Spickschen et al. [29] and Castagna et al. [32] pointed out that convertible stems had lower complication rates. These findings are 
coherent with our recent results and underline the importance of a shoulder arthroplasty system's modularity. To our knowledge, this study is the largest single-center trial with the longest follow-up after conversion from failed HA and TSA to RTSA. We are, however, aware of some limitations of this investigation. One is the smaller sample size in the retention group. This limitation may affect the analysis of confounding factors pertaining to variable indications for revision of hemiarthroplasties and total shoulder arthroplasties. However, there is no selection bias due to the retrospective study design, including criterium determining which group of the study the patients fell was driven by the type of the previously implanted prosthesis. This study's potential selection bias is that clinical outcome data from 29 patients (ten patients with complications, nineteen patients lost to followup) in the exchange group, and one patient (lost to follow-up) in the retention group were not available. We are also aware that the lack of personal follow-up and the limited sample size of the retention group patients might affect the results regarding patients' satisfaction and clinical outcome between the study groups, where no difference could be found. An additional obvious limitation is that operative time and blood loss were documented retrospectively from the anesthesia records.

\section{Conclusion}

Conversion of stemmed hemi- or total anatomical shoulder arthroplasty to reverse total shoulder arthroplasty with or without stem exchange leads to improved shoulder function and pain. Retention of the humeral stem reduces the surgical time, the intraoperative blood loss, and the risk of intra- and postoperative complications and reoperations without compromising mid- to long term functional outcomes.

\section{References}

1. Dilisio MF, Miller LR, Siegel EJ, Higgins LD. Conversion to reverse shoulder arthroplasty: Humeral stem retention vs. revision. Orthopedics. 2015;38(9):e773-9.

2. Kim SH, Wise BL, Zhang Y, Szabo RM. Increasing incidence of shoulder arthroplasty in the United States. J Bone Joint Surg Am. 2011;93(24):224954.

3. Knowles NK, Columbus MP, Wegmann K, Ferreira LM, Athwal GS, Revision shoulder arthroplasty: A systematic review and comparison of North American vs. European outcomes and complications. J Shoulder Elbow Surg. 2020;29(5):1071-82.

4. Scarlat MM. Complications with reverse total shoulder arthroplasty and recent evolutions. Int Orthop. 2013;37(5):843-51.

5. Zumstein MA, Pinedo M, Old J, Boileau P. Problems, complications, reoperations, and revisions in reverse total shoulder arthroplasty: A systematic review. J Shoulder Elbow Surg. 2011;20(1):146-57.

6. Besch L, Daniels-Wredenhagen M, Mueller M, Varoga D, Hilgert RE Seekamp A. Hemiarthroplasty of the shoulder after four-part fracture of the humeral head: A long-term analysis of 34 cases. J Trauma. 2009;66(1):211-4.

7. Cadet ER, Ahmad CS. Hemiarthroplasty for three- and four-part proximal humerus fractures. J Am Acad Orthop Surg. 2012;20(1):17-27.

8. Gartsman GM, Roddey TS, Hammerman SM. Shoulder arthroplasty with or without resurfacing of the glenoid in patients who have osteoarthritis. J Bone Joint Surg Am. 2000;82(1):26-34.

9. Lo IK, Litchfield RB, Griffin S, Faber K, Patterson SD, Kirkley A. Qualityof-life outcome following hemiarthroplasty or total shoulder arthroplasty in patients with osteoarthritis. A prospective, randomized trial. J Bone
Joint Surg Am. 2005;87(10):2178-85

10. Mighell MA, Kolm GP, Collinge CA, Frankle MA. Outcomes of hemiarthroplasty for fractures of the proximal humerus. J Shoulder Elbow Surg. 2003;12(6):569-77.

11. Robinson WA, Wagner ER, Cofield RH, Sanchez-Sotelo J, Sperling JW. Long-term outcomes of humeral head replacement for the treatment of osteoarthritis; a report of 44 arthroplasties with minimum 10-year followup. J Shoulder Elbow Surg. 2018;27(5):846-52.

12. Gohlke F, Rolf O. [Revision of failed fracture hemiarthroplasties to reverse total shoulder prosthesis through the transhumeral approach: method incorporating a pectoralis-major-pedicled bone window]. Oper Orthop Traumatol. 2007;19(2):185-208.

13. Holschen M, Franetzki B, Witt KA, Liem D, Steinbeck J. Conversions from anatomic shoulder replacements to reverse total shoulder arthroplasty: do the indications for initial surgery influence the clinical outcome after revision surgery? Arch Orthop Trauma Surg. 2017;137(2):167-72.

14. Holschen M, Franetzki B, Witt KA, Liem D, Steinbeck J. Is reverse total shoulder arthroplasty a feasible treatment option for failed shoulder arthroplasty? A retrospective study of 44 cases with special regards to stemless and stemmed primary implants. Musculoskelet Surg. 2017;101(2):173-80.

15. Levy JC, Virani N, Pupello D, Frankle M. Use of the reverse shoulder prosthesis for the treatment of failed hemiarthroplasty in patients with glenohumeral arthritis and rotator cuff deficiency. J Bone Joint Surg Br. 2007;89(2):189-95

16. Patel DN, Young B, Onyekwelu I, Zuckerman JD, Kwon YW. Reverse total shoulder arthroplasty for failed shoulder arthroplasty. J Shoulder Elbow Surg. 2012;21(11):1478-83

17. Wieser K, Borbas P, Ek ET, Meyer DC, Gerber C. Conversion of stemmed hemi- or total to reverse total shoulder arthroplasty: Advantages of a modular stem design. Clin Orthop Relat Res. 2015;473(2):651-60.

18. Bohsali KI, Wirth MA, Rockwood CA. Complications of total shoulder arthroplasty. J Bone Joint Surg Am. 2006;88(10):2279-92.

19. Flury MP, Frey P, Goldhahn J, Schwyzer HK, Simmen BR. Reverse shoulder arthroplasty as a salvage procedure for failed conventional shoulder replacement due to cuff failure--midterm results. Int Orthop. 2011;35(1):53-60.

20. Melis B, Bonnevialle N, Neyton L, Levigne C, Favard L, Walch G, et al. Glenoid loosening and failure in anatomical total shoulder arthroplasty: is revision with a reverse shoulder arthroplasty a reliable option? J Shoulder Elbow Surg. 2012;21(3):342-9.

21. Ortmaier R, Resch H, Matis N, Blocher M, Auffarth A, Mayer M, et al Reverse shoulder arthroplasty in revision of failed shoulder arthroplastyoutcome and follow-up. Int Orthop. 2013;37(1):67-75.

22. Walker M, Willis MP, Brooks JP, Pupello D, Mulieri PJ, Frankle MA The use of the reverse shoulder arthroplasty for treatment of failed total shoulder arthroplasty. J Shoulder Elbow Surg. 2012;21(4):514-22.

23. Dines JS, Fealy S, Strauss EJ, Allen A, Craig EV, Warren RF, et al. Outcomes analysis of revision total shoulder replacement. J Bone Joint Surg Am. 2006;88(7):1494-500

24. Constant CR, Gerber C, Emery RJ, Sojbjerg JO, Gohlke F, Boileau P. A review of the Constant score: Modifications and guidelines for its use. J Shoulder Elbow Surg. 2008;17(2):355-61.

25. Constant CR, Murley AH. A clinical method of functional assessment of the shoulder. Clin Orthop Relat Res. 1987;214:160-4.

26. Gilbart MK, Gerber C. Comparison of the subjective shoulder value and the Constant score. J Shoulder Elbow Surg. 2007;16(6):717-21.

27. Gerber C, Fuchs B, Hodler J. The results of repair of massive tears of the 
rotator cuff. J Bone Joint Surg Am. 2000;82(4):505-15.

28. Fucentese SF, Sutter R, Wolfensperger F, Jost B, Gerber C. Large metaphyseal volume hemiprostheses for complex fractures of the proximal humerus. J Shoulder Elbow Surg. 2014;23(3):427-33.

29. Weber-Spickschen TS, Alfke D, Agneskirchner JD. The use of a modular system to convert an anatomical total shoulder arthroplasty to a reverse shoulder arthroplasty: Clinical and radiological results. Bone Joint J. 2015;97-B(12):1662-7.

30. Merolla G, Wagner E, Sperling JW, Paladini P, Fabbri E, Porcellini G
Revision of failed shoulder hemiarthroplasty to reverse total arthroplasty: Analysis of 157 revision implants. J Shoulder Elbow Surg. 2018;27(1):75-

31. Farshad M, Gerber C. Reverse total shoulder arthroplasty-from the most to the least common complication. Int Orthop. 2010;34(8):1075-82.

32. Castagna A, Delcogliano M, de Caro F, Ziveri G, Borroni M, Gumina S, et al. Conversion of shoulder arthroplasty to reverse implants: Clinical and radiological results using a modular system. Int Orthop. 2013;37(7):1297305. 